\title{
Total Laryngopharyngectomy
}

National Cancer Institute

\section{Source}

National Cancer Institute. Total Laryngopharyngectomy. NCI Thesaurus. Code C150661.

Surgical removal of the entire larynx and pharynx. 